Artículo original

\title{
CARACTERIZACIÓN DE AGREGADOS DE CINCO CANTERAS DE LA PROVINCIA DE TACNA Y SU OPTIMACIÓN DE USO EN OBRAS DE CONSTRUCCIÓN
}

\author{
CHARACTERIZATION OF AGGREGATES OF FIVE QUARRIES OF THE \\ PROVINCE OF TACNA AND ITS OPTIMIZATION FOR USE IN CONSTRUCTION \\ WORKS \\ Jesús Raúl Zavaleta Villanueva ${ }^{1}$ \\ Giovani Alonso Reátegui García ${ }^{2}$ \\ María Etelvina Duarte Lizarzaburu ${ }^{3}$
}

Información del artículo:

Recibido: $13 / 08 / 2020$

Aceptado: 7/12/2020

${ }^{1,2}$ Ingeniero Civil Universidad Privada de Tacna, Perú

${ }^{3}$ Docente Escuela de Ingeniería Civil, Universidad Privada de Tacna, Perú E-mail: 1jzava20@hotmail.com, ${ }^{2}$ giovani.reategui1@gmail.com, ${ }^{3}$ mduartel@gmail.com 


\section{Resumen}

La caracterización de los agregados de las cinco principales canteras de la provincia de Tacna que permita optimizar su uso en obras de construcción. Estudio de tipo cuantitativo, explicativa y descriptiva donde se determinó las propiedades físicas del agregado realizando los ensayos según los parámetros establecidos en la Norma Técnica Peruana NTP 400.037. Se elaboró el diseño de mezcla según el método $\mathrm{ACl}$ para $\mathrm{f}^{\prime} \mathrm{c}=210 \mathrm{~kg} / \mathrm{cm} 2$. Posterior a ello se realizaron nueve testigos de concreto, para cada de las siguientes canteras; Arunta, Magollo, Arunta II, Jonas I y la propuesta para explotación ubicada en el distrito de Sama, a edades de 7, 14 y 28 días. Al realizar el ensayo de compresión se observó que el concreto realizado con los diferentes agregados cumple con los parámetros establecidos por la Norma Técnica Peruana. La cantera propuesta ubicada en el distrito de Sama, mostró óptimas condiciones para la explotación de agregados, ya que al realizar el ensayo de compresión obtuvo una resistencia de $319,61 \mathrm{~kg} / \mathrm{cm} 2$ a 28 días.

Palabras Clave: Canteras; Diseño de Mezcla; Resistencia a la compresión.

\section{Abstract}

The characterization of the aggregates of the five main quarries of the province of Tacna that allows to optimize its use in construction works. Quantitative, explanatory and descriptive study where the physical properties of the aggregate were determined by performing the tests according to the parameters established in the Peruvian Technical Standard NTP 400.037. The mixing design was developed according to the ACl method for f'c$210 \mathrm{~kg} / \mathrm{cm} 2$. Subsequently, nine concrete witnesses were made, for each of the following quarries; Arunta, Magollo, Arunta II, Jonas I and the proposal for exploitation located in the district of Sama, at ages 7, 14 and 28 days. When performing the compression test it was observed that the concrete made with the different aggregates complies with the parameters established by the Peruvian Technical Standard. The proposed quarry located in Sama district showed optimal conditions for the exploitation of aggregates, since when performing the compression test it obtained a resistance of $319.61 \mathrm{~kg} / \mathrm{cm} 2$ to 28 days.

Keywords: Quarries; Mixture Design: Compressive Strength. 


\section{Introducción}

Actualmente se ejecutan obras de construcción empleando agregados de diversas canteras, no obstante, algunos constructores que requieren del material, emplean sin comprender sus propiedades, en consecuencia esto produce un alto grado de vacilación al instante de producir el concreto, ya que al desconocer las propiedades de sus componentes, no es posible saber si este logrará la resistencia requerida según el diseño. El origen del agregado es importante para determinar la calidad del mismo, en tal sentido, los agregados de origen aluvial o tipo sedimentario poseen gran resistencia al desgaste, aspecto relevante para el consumidor.

Con la extracción de los agregados, no solo se logra abastecer de material al mercado, sino que además se deben cumplir los requisitos de calidad, por lo que en la extracción se debe evaluar el diseño de la muestra extraída para controlar la demanda del mercado, asimismo las propiedades del agregado para su uso en el concreto.

Ya que la extracción de grava y bancos de arena en el país se lleva a cabo con un mínimo y a veces ningún control de calidad, no se asegura que el material obtenido cumpla con los requisitos de las Normas técnicas empleadas. Respecto a estudios similares, varios autores (Torres, 2013; Diaz et al., 2014; Orozco y palacio 2015) han realizado trabajos para establecer la resistencia a la compresión que fue calculada entre 7 y 14 días, otros estudios responden a caracterizar el concreto que resulta de agregar o modificar los componentes de la mezcla (Arévalo, 2016; Piraban y Farfán, 2018, Chanini 2015; Mamani y salinas, 2015) y establecer si esas modificaciones son adecuadas para formar concretos de buena calidad, en esa dirección Castillo (2009), indica que las propiedades del concreto depende de directamente de los elementos que se utilicen en su fabricación y de la cantidad que se empleen en este proceso, siendo algunas de sus propiedades la trabajabilidad y consistencia; segregación; resistencia; exudación y durabilidad, mientras que La norma NTP 400.037 Normas Técnicas Peruanas (NTP) y A.S.T.M. "American Society of Testing Materials" establecen las características y los procedimientos que deberán cumplirse en los agregados al momento de ser utilizados y recomienda hacer los siguientes ensayos: granulometría, peso específico; absorción y humedad y peso unitario.

\section{Objetivos}

La investigación desarrolla el estudio de las principales canteras que se explotan la ciudad de Tacna, tomando en cuenta las Normas ASTM y las NTP correspondientes.

\section{Metodología}

Se realizó muestreos en diferentes canteras que abastecen de material de construcción, en principal los agregados para obras civiles en la provincia de Tacna, agregados finos (Arena gruesa) y Agregado grueso (Piedra chancada $3 / 4$ "). Las canteras que participaron del estudio fueron Arunta, Jonas I, Arunta II, Magollo y Sama que están ubicadas en la región Tacna. El material extraído se 
encuentran depositados en grandes bancos, siendo este retirado de la parte inferior, la de la parte intermedia y la parte superior de las pilas de agregado $s$, con la finalidad de obtener un buen muestreo, así como establece la norma técnica peruana NTP 400.010:2016, que explica las recomendaciones para lograr una muestra más uniforme. Para ejecutar los ensayos físicos, químicos y mecánicos al material extraído de cantera (muestreo de agregados) se tuvieron que embolsar en sacos de plástico de $50 \mathrm{~kg}$ de capacidad a cada uno.

Asimismo, una vez culminada la realización de los ensayos, se procedió a realizar una segunda evaluación a aquellos agregados que no cumplieron con los parámetros establecidos en la NTP 400.037.2014, para poder conseguir un superior diseño de mezcla al optimizar las características de los agregados.

Los ensayos de humedad se realizaron tomando como referencia la Norma NTP 339.185:2013 (Revisión 2018), el Peso Específico y Absorción del Agregado Fino bajo la Norma NTP 400.022:2013, mientras que el Peso Específico y Absorción del Agregado Grueso bajo la Norma NTP 400.021:2013, el Peso Unitario y Contenido de Vacíos bajo la Norma NTP 400.017:2011, la Granulometría por tamizado bajo la Norma NTP 400.012:2013, Cantidad de finos bajo la Norma NTP 400.018:2013

Para el diseño de mezclas se consideró en la dosificación del concreto, la característica de los materiales, en específico para el cemento; Densidad (Gc), Masa unitaria suelta (MUSc), densidad de agua (Ga) se asume $\mathrm{Ga}=1,00 \mathrm{~kg} / \mathrm{dm} 3$. En agregados los análisis granulométrico de los agregados incluyendo el cálculo del módulo de finura (MF) o del tamaño máximo nominal (TMN), según el árido, Densidad aparente seca $(\mathrm{G})$ y porcentaje de absorción de los agregados (\% ABS.). También se el porcentaje de humedad de los agregados inmediatamente antes de hacer las mezclas (Wn). Masas unitarias sueltas (MUS). En el caso de insumos se considera la densidad (Gad)

Las características geométricas y de diseño del elemento o elementos estructurales a construir, y condiciones de colocación de la mezcla considerados son consistencia apropiada, Chequeo del tamaño máximo nominal. Resistencia de diseño del calculista ( $F^{\prime} c$ o F'r), Condiciones de exposición de la estructura. De acuerdo con ellas, podrá obtenerse la máxima relación agua/cemento que puede utilizarse en el proporcionamiento de la mezcla.

Por otro lado los pasos a seguir para el diseño de mezcla de $\mathrm{fc}=210 \mathrm{~kg} / \mathrm{cm} 2$, con la finalidad de obtener las proporciones de la mezcla del concreto que cumpla las características deseadas, se preparó una primera mezcla de prueba, teniendo como base unas proporciones iniciales que se determinan siguiendo el orden siguiente; Selección del asentamiento, chequeo del tamaño máximo nominal, estimación del agua de mezcla, determinación de la resistencia de dosificación, selección de la relación Agua/Cemento, cálculo del contenido de cemento, cálculo de la cantidad de cada agregado, cálculo de proporciones iniciales, primera mezcla de prueba y ajuste por humedad de los agregados

Con los resultados de la primera mezcla se procedió a ajustar las proporciones para que cumpla con el asentamiento deseado y el grado de manejabilidad requerido, posteriormente se prepara una segunda mezcla de prueba con las proporciones ajustadas; las propiedades de ésta segunda mezcla se comparan con las exigidas y si difieren se reajustan nuevamente. Se prepara una tercera mezcla de prueba que debe cumplir con el asentamiento y la resistencia deseada; en caso que no cumpla alguna de las condiciones por errores cometidos o debido a la aleatoriedad misma de 
los ensayos, se puede continuar haciendo ajustes semejantes a los indicados hasta obtener los resultados esperados.

\section{Resultados}

\section{Ensayos físicos}

El contenido de humedad más alto de la arena que se obtuvo en las diferentes canteras fue en la cantera Arunta II y en cuanto al alto contenido de humedad de la arena y piedra se obtuvo en la cantera de Sama debido a que presenta garuas y neblinas que se dan en la madrugada como en temporadas de invierno.

Un agregado grueso es de buena calidad si presenta una absorción menor a 3\% y en el caso de los agregados finos menor a 5\%. Una absorción baja junto con una forma adecuada (partículas aproximadamente redondas). Por otro lado, la absorción del agregado estaba directamente relacionada con la alteración del mismo y la durabilidad de estructuras, haciéndolos más resistentes ante procesos de congelamiento o deshielo.

Los pesos específicos de los agregados en todas las canteras mostraron resultados dentro de los parámetros establecidos resaltando que la cantera Arunta II mostró resultados casi similares debido al tamaño de la piedra que mostraba, se puede observar que la cantera Magollo muestra pesos específicos ideales y más resaltantes a comparación de las demás canteras así mismo la cantera Sama muestra pesos específicos bajos, pero dentro de los límites aceptables para la elaboración de diseño de mezcla

Los datos de los pesos unitarios muestran uniformidad en la arena como en la piedra en todas las canteras, manteniendo los resultados dentro de los parámetros establecidos en la norma técnica peruana, con respecto al grafico se puede observar que todas las canteras muestran buenos resultados en el peso unitario suelto teniendo como resultado promedio de $1,50 \mathrm{gr} / \mathrm{cc}$ en la arena y de $1,40 \mathrm{gr} / \mathrm{cc}$ para la piedra.

El peso unitario compactado de la piedra mostro uniformidad en las canteras ubicadas cerca de la ciudad de Tacna en cuanto a la cantera, ubicada en sama un peso unitario compactado óptimo en la arena y piedra teniendo como resultado promedio de $1,60 \mathrm{gr} / \mathrm{cc}$ en la arena y de 1,50 gr/cc para la piedra.

Podemos establecer que los módulos de finura de los agregados empleados en concreto no deben ser menores de 2,3 ni mayores a 3,1; lo cual se aprecia en el cuadro que cumplen con lo establecido. 
Tabla 1

Resumen de parámetros analizados

\begin{tabular}{|c|c|c|c|c|c|c|c|c|c|c|}
\hline \multirow[t]{2}{*}{ Parámetros } & \multicolumn{2}{|c|}{ Arunta II } & \multicolumn{2}{|c|}{ Jonas I } & \multicolumn{2}{|c|}{ Arunta } & \multicolumn{2}{|c|}{ Magollo } & \multicolumn{2}{|c|}{ Sama } \\
\hline & Grueso & Fino & Grueso & Fino & Grueso & Fino & Grueso & Fino & Grueso & Fino \\
\hline Humedad (\%) & 0,15 & 2,50 & 0,25 & 2,00 & 0,15 & 1,04 & 0,13 & 0,84 & 0,54 & 0,77 \\
\hline Absorción (\%) & 2,25 & 1,27 & 1,45 & 1,50 & 1,40 & 1,62 & 1,52 & 1,70 & 1,55 & 1,85 \\
\hline \multicolumn{11}{|l|}{ Peso Específico } \\
\hline (gr/cc) & 2,60 & 2,61 & 2,60 & 2,46 & 2,57 & 2,66 & 2,55 & 2,72 & 2,48 & 2,42 \\
\hline \multicolumn{11}{|l|}{ Peso unitario } \\
\hline suelto(gr/cc) & 1,50 & 1,56 & 1,45 & 1,66 & 1,33 & 1,71 & 1,31 & 1,75 & 1,52 & 1,62 \\
\hline \multicolumn{11}{|l|}{ Peso unitario } \\
\hline compactado (gr/cc) & 1,68 & 1,77 & 1,60 & 1,80 & 1,47 & 1,92 & 1,46 & 1,93 & 1,69 & 1,79 \\
\hline Módulo de finura & - & 3,10 & - & 3,00 & - & 3,00 & - & 2,80 & - & 3,00 \\
\hline \multicolumn{11}{|l|}{ Tamaño máx. del } \\
\hline agregado (pulg.) & $3 / 4 "$ & - & $3 / 4 "$ & - & 3/4" & - & $3 / 4 "$ & - & $3 / 4 "$ & - \\
\hline Asentamiento (pulg) & \multicolumn{2}{|c|}{ 3"- 4" } & \multicolumn{2}{|c|}{ 3"- 4" } & \multicolumn{2}{|c|}{ 3"- 4" } & \multicolumn{2}{|c|}{ 3"- 4" } & \multicolumn{2}{|c|}{ 3"- 4" } \\
\hline
\end{tabular}

Nota. Elaboración propia.

\section{Diseños de mezcla}

Las proporciones para el diseño de la mezcla de concreto cumplieron con las características con los materiales disponibles, se logró mediante el sistema de prueba de ajuste o corrección por humedad y absorción. Se realizó el diseño por el método $\mathrm{ACl}$ para una resistencia de $210 \mathrm{~kg} / \mathrm{cm} 2$, a continuación, se muestran los diferentes diseños de mezcla de cada cantera en las tablas 2 , tabla 3 , tabla, tabla 5 y tabla 6. 
Tabla 2

Diseño de mezcla-Cantera Arunta

\begin{tabular}{|c|c|c|c|}
\hline Características del Agregado & Unidad & Agregado Grueso & Agregado Fino \\
\hline Peso Especifico & $\mathrm{g} / \mathrm{cc}$ & 2,57 & 2,66 \\
\hline Peso Unitario Suelto & $\mathrm{g} / \mathrm{cc}$ & 1,33 & 1,71 \\
\hline Peso Unitario Varillado o Compactado & $\mathrm{g} / \mathrm{cc}$ & 1,47 & 1,92 \\
\hline Tamaño Máximo Nominal & pulg. & $3 / 4^{\prime \prime}$ & - \\
\hline Módulo de Fineza & pulg. & - & 3,10 \\
\hline Humedad Absorción & $\%$ & 1,40 & 1,62 \\
\hline Humedad Natural & $\%$ & 0,15 & 1,05 \\
\hline Resistencia a la Compresión & $\mathrm{Kg} / \mathrm{cm}^{2}$ & - & 210 \\
\hline $\begin{array}{l}\text { Peso Específico del Cemento } \\
\text { Yura (Tipo IP) }\end{array}$ & $\mathrm{g} / \mathrm{cc}$ & - & 2,85 \\
\hline Peso Específico del Agua $(\mathrm{H} 2 \mathrm{O})$ & $\mathrm{g} / \mathrm{cc}$ & - & 1,00 \\
\hline
\end{tabular}

\begin{tabular}{|c|c|c|c|}
\hline Slump : 3" a 4" & \multicolumn{3}{|c|}{$F^{\prime} \mathrm{cr}: 294 \mathrm{Kg} / \mathrm{cm} 2$} \\
\hline Agua $\quad: 200$ & \multicolumn{3}{|c|}{ Relación de Agua/Cemento: 0,55 } \\
\hline Aire $\quad: 2.0$ & \multicolumn{3}{|c|}{ Volumen del Agregado Grueso (b/bo):0,59 } \\
\hline Materiales para $1 \mathrm{~m}^{3}$ & P.E. & Peso (Kg) & Volumen $\left(1 \mathrm{~m}^{3}\right)$ \\
\hline Agua & 1000 & 200,00 & 0,200 \\
\hline Cemento & 2850 & 363,64 & 0,128 \\
\hline Aire & - & - & 0,020 \\
\hline Piedra & 2570 & 867,30 & 0,337 \\
\hline Arena & 2660 & 837,73 & 0,315 \\
\hline Materiales para $1 \mathrm{~m}^{3}$ & Corrección por humedad y absorción & Peso (Kg) & Volumen $\left(1 \mathrm{~m}^{3}\right)$ \\
\hline Agua & 1000 & 215,00 & 0,216 \\
\hline Cemento & 2850 & 363,64 & 0,128 \\
\hline Aire & - & - & 0,020 \\
\hline Piedra & 2570 & 868,60 & 0,338 \\
\hline Arena & $\begin{array}{c}2660 \\
\text { Dosificación }\end{array}$ & 846,53 & 0,318 \\
\hline Materiales & Peso & Volumen & 1 bolsa cemento \\
\hline Cemento & 1,00 & 1,00 & 42,50 \\
\hline Aire & 2,33 & 2,49 & 98,94 \\
\hline Piedra & 2,39 & 2,65 & 101,52 \\
\hline Arena & 0,59 & 1,69 & 25,20 \\
\hline Materiales (Kg) & Cemento & Arena & Piedra \\
\hline Una Probeta Agua $(L)=1,14$ & 1,93 & 4,49 & 4,60 \\
\hline
\end{tabular}

Nota. Elaboración propia. 
Tabla 3

Diseño de mezcla-Cantera Jonas I

\begin{tabular}{|c|c|c|c|}
\hline Características del Agregado & Unidad & Agregado Grueso & Agregado Fino \\
\hline Peso Especifico & $\mathrm{g} / \mathrm{cc}$ & 2,60 & 2,46 \\
\hline Peso Unitario Suelto & $\mathrm{g} / \mathrm{cc}$ & 1,45 & 1,66 \\
\hline Peso Unitario Varillado o & $\mathrm{g} / \mathrm{cc}$ & 1,60 & 1,80 \\
\hline \multicolumn{4}{|l|}{ Compactado } \\
\hline Tamaño Máximo Nominal & pulg. & $3 / 4 "$ & - \\
\hline Módulo de Fineza & pulg. & - & 3,00 \\
\hline Humedad Absorción & $\%$ & 1,45 & 1,50 \\
\hline Humedad Natural & $\%$ & 0,25 & 2,00 \\
\hline Resistencia a la Compresión & $\mathrm{Kg} / \mathrm{cm}^{2}$ & & 210 \\
\hline Peso Específico del Cemento & $\mathrm{g} / \mathrm{cc}$ & & 2,85 \\
\hline \multicolumn{4}{|l|}{ Yura (Tipo IP) } \\
\hline Peso Específico del Agua(H2O) & $\mathrm{g} / \mathrm{cc}$ & & 1,00 \\
\hline
\end{tabular}

Consideraciones

\begin{tabular}{|c|c|c|c|}
\hline Slump : $3^{\prime \prime}$ a $4^{\prime \prime}$ & \multicolumn{3}{|c|}{$\mathrm{F}^{\prime} \mathrm{cr}: 294 \mathrm{Kg} / \mathrm{cm}^{2}$} \\
\hline Agua $\quad: 200$ & \multicolumn{3}{|c|}{ Relación de Agua/Cemento: 0,55 } \\
\hline$: 2,0$ & \multicolumn{3}{|c|}{ Volumen del Agregado Grueso (b/bo):0,60 } \\
\hline Materiales para $1 \mathrm{~m}^{3}$ & P.E. & Peso (kg) & Volumen(1m3) \\
\hline Agua & 1000 & 200,00 & 0,200 \\
\hline Cemento & 2850 & 363,64 & 0,128 \\
\hline Aire & - & - & 0,020 \\
\hline Piedra & 2600 & 960,00 & 0,369 \\
\hline Arena & 2460 & 696,62 & 0,283 \\
\hline
\end{tabular}

Corrección por humedad y absorción

\begin{tabular}{|c|c|c|c|}
\hline Materiales para $1 \mathrm{~m}^{3}$ & P.E. & PESO (Kg) & Volumen $\left(1 \mathrm{~m}^{3}\right)$ \\
\hline Agua & 1000 & 208,04 & 0,208 \\
\hline Cemento & 2850 & 363,64 & 0,128 \\
\hline Aire & - & - & 0,020 \\
\hline Piedra & 2600 & 962,40 & 0,370 \\
\hline \multirow[t]{2}{*}{ Arena } & 2460 & 710,55 & 0,289 \\
\hline & \multicolumn{2}{|c|}{ Dosificación } & \\
\hline Materiales & Peso & Volumen & 1 bolsa de cemento \\
\hline Cemento & 1,00 & 1,00 & 42,50 \\
\hline Aire & 1,95 & 2,26 & 83,05 \\
\hline Piedra & 2,65 & 2,90 & 112,48 \\
\hline Arena & 0,57 & 1,63 & 24,31 \\
\hline Materiales (kg) & Cemento & Arena & Piedra \\
\hline Una Probeta Agua $(\mathrm{L})=1,14$ & 1,93 & 3,77 & 5,10 \\
\hline
\end{tabular}

Nota. Elaboración propia. 
Tabla 4

Diseño de mezcla-Cantera Arunta II

\begin{tabular}{|c|c|c|c|}
\hline Características del agregado & Unidad & Agregado grueso & Agregado fino \\
\hline Peso Especifico & $\mathrm{g} / \mathrm{cc}$ & 2,60 & 2,61 \\
\hline Peso Unitario Suelto & $\mathrm{g} / \mathrm{cc}$ & 1,50 & 1,56 \\
\hline Peso Unitario Varillado o & $\mathrm{g} / \mathrm{cc}$ & 1,68 & 1,77 \\
\hline \multicolumn{4}{|l|}{ Compactado } \\
\hline Tamaño Máximo Nominal & pulg. & $3 / 4 "$ & - \\
\hline Módulo de Fineza & pulg. & - & 3,10 \\
\hline Humedad Absorción & $\%$ & 2,25 & 1,27 \\
\hline Humedad Natural & $\%$ & 0,15 & 2,50 \\
\hline Resistencia a la Compresión & \multicolumn{2}{|l|}{$\mathrm{Kg} / \mathrm{cm}^{2}$} & 210 \\
\hline \multicolumn{2}{|l|}{ Yura (Tipo IP) } & & \\
\hline Peso Específico del Agua(H2O) & \multicolumn{2}{|l|}{$\mathrm{g} / \mathrm{cc}$} & 1,00 \\
\hline \multicolumn{4}{|l|}{ Consideraciones } \\
\hline Slump : 3" a 4" & \multicolumn{3}{|l|}{$F^{\prime} \mathrm{cr}: 294 \mathrm{Kg} / \mathrm{cm}^{2}$} \\
\hline Agua : 200 & \multicolumn{3}{|c|}{ Relación de Agua/Cemento: 0,55 } \\
\hline Aire $: 2,0$ & \multicolumn{3}{|c|}{ Volumen del Agregado Grueso (b/bo):0,56 } \\
\hline Materiales para $1 \mathrm{~m}^{3}$ & P.E. & Peso $(\mathrm{Kg})$ & Volumen $\left(1 \mathrm{~m}^{3}\right)$ \\
\hline Agua & 1000 & 200,00 & 0,200 \\
\hline Cemento & 2850 & 363,64 & 0,128 \\
\hline Aire & - & - & 0,020 \\
\hline Piedra & 2600 & 940,80 & 0,362 \\
\hline Arena & 2610 & 758,37 & 0,291 \\
\hline \multicolumn{4}{|c|}{ Corrección por humedad y absorción } \\
\hline Materiales para $1 \mathrm{~m}^{3}$ & P.E. & Peso (kg) & Volumen $\left(1 \mathrm{~m}^{3}\right)$ \\
\hline Agua & 1000 & 210,43 & 0,210 \\
\hline Cemento & 2850 & 363,64 & 0,128 \\
\hline Aire & - & - & 0,020 \\
\hline Piedra & 2600 & 942,21 & 0,362 \\
\hline Arena & 2610 & 777,33 & 0,298 \\
\hline & Dosificacic & & \\
\hline Materiales & Peso & Volumen & 1 bolsa de cemento \\
\hline Cemento & 1,00 & 1,00 & 42,50 \\
\hline Aire & 2,14 & 2,33 & 90,85 \\
\hline Piedra & 2,59 & 2,84 & 110,12 \\
\hline Arena & 0,58 & 1,65 & 24,59 \\
\hline Materiales (kg) & Cemento & Arena & Piedra \\
\hline Una Probeta Agua $(L)=1,12$ & 1,93 & 4,12 & 5,00 \\
\hline
\end{tabular}

Nota. Elaboración propia. 
Tabla 5

Diseño de mezcla-Cantera Magollo

\begin{tabular}{|c|c|c|c|}
\hline Características del Agregado & Unidad & Agregado grueso & Agregado Fino \\
\hline Peso Especifico & $\mathrm{g} / \mathrm{cc}$ & 2,55 & 2,72 \\
\hline Peso Unitario Suelto & $\mathrm{g} / \mathrm{cc}$ & 1,31 & 1,75 \\
\hline Peso Unitario Varillado o & $\mathrm{g} / \mathrm{cc}$ & 1,46 & 1,93 \\
\hline \multicolumn{4}{|l|}{ Compactado } \\
\hline Tamaño Máximo Nominal & pulg. & $3 / 4 "$ & - \\
\hline Módulo de Fineza & pulg. & - & 2,80 \\
\hline Humedad Absorción & $\%$ & 1,52 & 1,70 \\
\hline Humedad Natural & $\%$ & 0,13 & 0,84 \\
\hline Resistencia a la Compresión & $\mathrm{Kg} / \mathrm{cm}^{2}$ & \multicolumn{2}{|r|}{210} \\
\hline Peso Específico del Cemento & $\mathrm{g} / \mathrm{cc}$ & \multicolumn{2}{|r|}{2,85} \\
\hline \multicolumn{4}{|l|}{ Yura (Tipo IP) } \\
\hline Peso Específico del Agua(H2O) & $\mathrm{g} / \mathrm{cc}$ & \multicolumn{2}{|r|}{1,00} \\
\hline
\end{tabular}

\section{Consideraciones}

Slump : 3" a 4"

Agua : 200

Aire $: 2,0$

Materiales para $1 \mathrm{~m}^{3}$

$F^{\prime} \mathrm{cr}: 294 \mathrm{Kg} / \mathrm{cm}^{2}$

Relación de Agua/Cemento: 0,55

Volumen del Agregado Grueso (b/bo): 0,62

Agua

P.E.

Peso (kg)

Volumen $\left(1 \mathrm{~m}^{3}\right)$

Cemento

1000

200.00

0,200

2850

363,64

0,128

Aire

$-$

Piedra

2550

05,20

0,020

Arena

2720

809,00

0,355

0,297

Corrección por humedad y absorción

\begin{tabular}{|c|c|c|c|}
\hline \multirow[b]{2}{*}{ Materiales para $1 \mathrm{~m}^{3}$} & & \multirow[b]{2}{*}{ Volumen $\left(1 \mathrm{~m}^{3}\right)$} \\
\hline & P.E. & Peso (kg) & \\
\hline Agua & 1000 & 219,54 & 0,220 \\
\hline Cemento & 2850 & 363,64 & 0,128 \\
\hline Aire & - & - & 0,020 \\
\hline Piedra & 2550 & 906,38 & 0,355 \\
\hline Arena & 2720 & 815,80 & 0,300 \\
\hline \multicolumn{4}{|c|}{ Dosificación } \\
\hline Materiales & Peso & Volumen & 1 bolsa de cemento \\
\hline Cemento & 1,00 & 1,00 & 42,50 \\
\hline Aire & 2,14 & 2,35 & 95,35 \\
\hline Piedra & 2,49 & 2,79 & 105,93 \\
\hline Arena & 0,60 & 1,72 & 25,66 \\
\hline Materiales (kg) & Cemento & Arena & Piedra \\
\hline Una Probeta Agua $(\mathrm{L})=1,16$ & 1,93 & 4,32 & 4,81 \\
\hline
\end{tabular}

Nota. Elaboración propia. 
Tabla 6

Diseño de mezcla-Cantera Sama

\begin{tabular}{|c|c|c|c|}
\hline Características del agregado & Unidad & Agregado grueso & Agregado fino \\
\hline Peso Especifico & $\mathrm{g} / \mathrm{cc}$ & 2,48 & 2,42 \\
\hline Peso Unitario Suelto & $\mathrm{g} / \mathrm{cc}$ & 1,52 & 1,62 \\
\hline $\begin{array}{l}\text { Peso Unitario Varillado o } \\
\text { Compactado }\end{array}$ & $\mathrm{g} / \mathrm{cc}$ & 1,69 & 1,79 \\
\hline Tamaño Máximo Nominal & pulg. & $3 / 4 "$ & - \\
\hline Módulo de Fineza & pulg. & - & 3,00 \\
\hline Humedad Absorción & $\%$ & 1,55 & 1,85 \\
\hline Humedad Natural & $\%$ & 0,54 & 0,78 \\
\hline Resistencia a la Compresión & $\mathrm{Kg} / \mathrm{cm} 2$ & \multicolumn{2}{|c|}{210} \\
\hline Peso Específico del Cemento & $\mathrm{g} / \mathrm{cc}$ & \multirow{2}{*}{\multicolumn{2}{|c|}{2,85}} \\
\hline Yura (Tipo IP) & & & \\
\hline Peso Específico del Agua(H2O) & $\mathrm{g} / \mathrm{cc}$ & \multicolumn{2}{|c|}{1,00} \\
\hline
\end{tabular}

$\mathrm{F}^{\prime} \mathrm{cr}: 294 \mathrm{Kg} / \mathrm{cm}^{2}$

Agua : 200

Relación de Agua/Cemento: 0,55

Aire : 2.0

Volumen del Agregado Grueso (b/bo):0,60

Materiales para $1 \mathrm{~m}^{3}$

Agua

P.E.

Peso (kg)

Volumen(1m3)

1000

200,00

0,200

Cemento

2850

363,64

0,128

Aire

Piedra

2480

1014,00

0,020

Arena

2420

589,36

0,409

0,244

Corrección por humedad y absorción

\begin{tabular}{lccc} 
Materiales para $\mathbf{1 \mathbf { m } ^ { 3 }}$ & P.E. & Peso (kg) & Volumen (1m3) \\
Agua & 1000 & 216,55 & 0,217 \\
Cemento & 2850 & 363,64 & 0,128 \\
Aire & - & - & 0,020 \\
Piedra & 2480 & 1019,48 & 0,411 \\
Arena & 2420 & 593,96 & 0,245 \\
Materiales & Dosificación & & Volumen \\
Cemento & Peso & $\mathbf{1}$ Bolsa de Cemento \\
Aire & 1,00 & 1,00 & 42,50 \\
Piedra & 1,63 & 1,92 & 69,42 \\
Arena & 2,80 & 3,22 & 119,15 \\
Materiales (kg) & 0,60 & 1,70 & 25,31 \\
Una Probeta Agua (L)=1,15 & Cemento & Arena & Piedra \\
\hline
\end{tabular}

Nota. Elaboración propia. 


\section{Ensayo de compresión de probetas de concreto}

La resistencia más alta a los 14 días que se obtuvo en las diferentes canteras fue en la cantera Sama $\left(194,37 \mathrm{~kg} / \mathrm{cm}^{2}\right)$ y en cuanto a la resistencia más baja se obtuvo en la cantera de Magollo $\left(194,97 \mathrm{~kg} / \mathrm{cm}^{2}\right)$

\section{Figura 1}

Ensayo de Compresión de Probetas de concreto a los 7 días

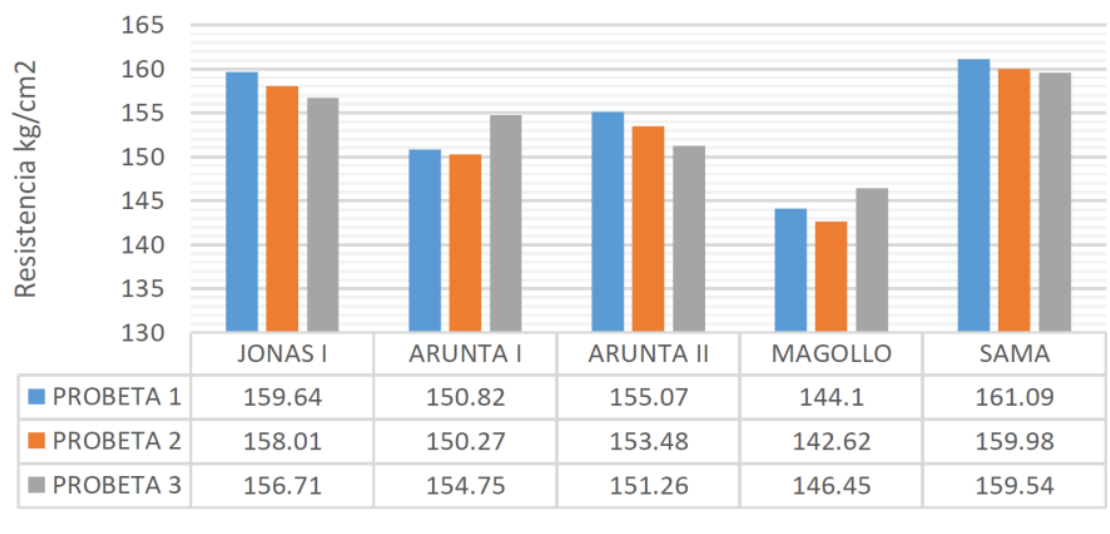

- PROBETA 1 PROBETA 2 - PROBETA 3

Nota. Elaboración propia.

La resistencia más alta a los 14 días que se obtuvo en las diferentes canteras fue en la cantera Sama $\left(194,37 \mathrm{~kg} / \mathrm{cm}^{2}\right)$ y en cuanto a la resistencia más baja se obtuvo en la cantera de Magollo $\left(194,97 \mathrm{~kg} / \mathrm{cm}^{2}\right)$.

\section{Figura 2}

Ensayo de Compresión de Probetas de concreto a los 14 días

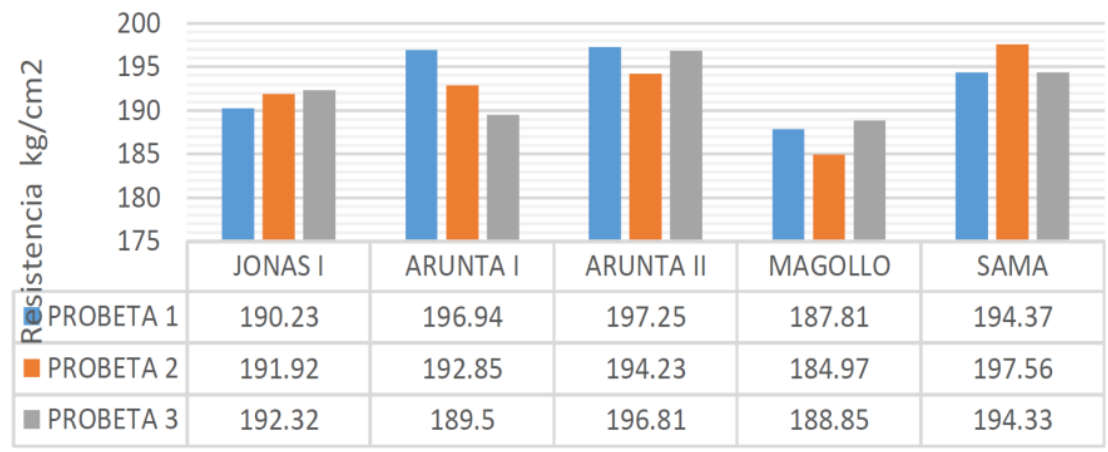

-PROBETA 1 -PROBETA 2 -PROBETA 3

Nota. Elaboración propia. 
La resistencia más alta a los 28 días que se obtuvo en las diferentes canteras fue en la cantera Jonas I $\left(322,29 \mathrm{~kg} / \mathrm{cm}^{2}\right)$ y en cuanto a la resistencia más baja se obtuvo en la cantera de Magollo $\left(311,54 \mathrm{~kg} / \mathrm{cm}^{2}\right)$.

\section{Figura 3}

Ensayo de Compresión de Probetas de concreto a los 28 días

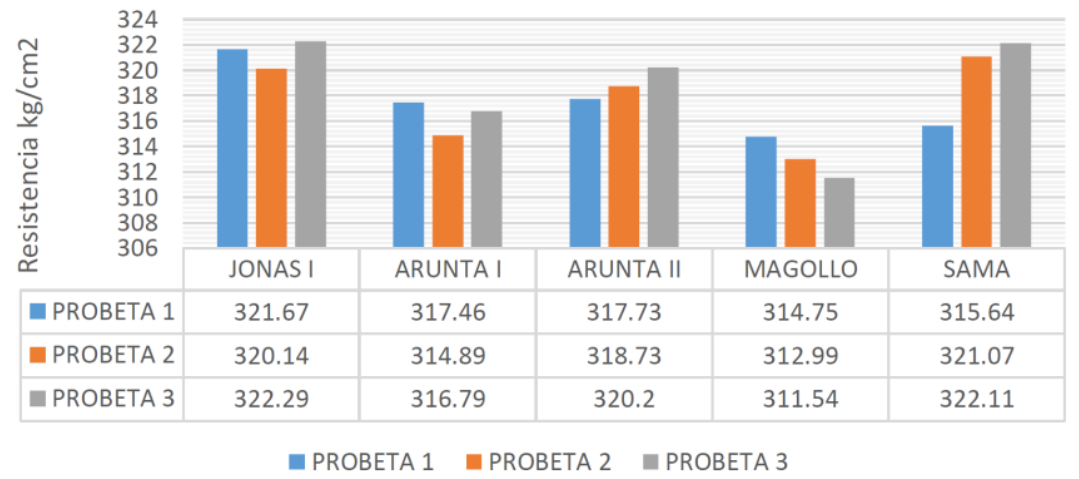

Nota. Elaboración propia.

\section{Discusión}

En la evaluación de los agregados mediante la realización de ensayos, se determinó que cumplen lo permitido por la NTP 400.037:2014, en la elaboración de un concreto convencional, motivo por el cual no se realizó otras evaluaciones, siendo las muestras usadas in situ sin adulteración, cumpliéndose con los límites necesarios y la optimización de los resultados para el diseño de un concreto.

En referencia a las características del agregado fino, no se eliminó los limos o arcillas, debido a que no excedían el límite de 5 \% según lo contemplado en la norma NTP 400.037:2014. En el caso del agregado grueso se ejecutó en análisis granulométrico, encontrándose el tamaño máximo nominal de $3 / 4^{\prime \prime}$, así mismo se precisa que los valores de los materiales ensayados, presentan desviaciones estándar adecuadas.

Las características de los parámetros de la cantera de Sama resultaron aceptables comprados con la NTP, asiendo aceptables y similares a las canteras existentes, respecto a su funcionamiento se puede afirmar que son aptas para la utilización y fabricación de concretos.

\section{Contenido de humedad}

Respecto a los ensayos de humedad, el agregado grueso presenta 0,54 \% de humedad para la cantera de Sama, mientras que Jonas I presentan 0,25 \%, por otro lado Magollo y Arunta presentan valores cercanos a 0,15\%, siendo las únicas que mantienen el mismo valor para el agregado grueso (piedra $3 / 4$ ), todo lo contrario ocurre con las canteras Arunta II y Jonas I quienes presentan altos valores de humedad en arena y piedra, ganando humedad en los dos tipos de 
agregados (fino y grueso), por lo tanto el aporte de agua en el diseño de mezcla, es mucho mayor en las canteras Arunta II y Jonas I debido a su contenido de humedad.

Al no tener un límite para el contenido de humedad en los materiales como los agregados, se debe tener en cuenta que no deben ser menores a los de la absorción, ya que si eso sucediera, se deberá agregar más agua al concreto para compensar la cantidad de agua que lograran absorber los agregados empleados. Por el contrario si la humedad es mayor que la absorción, tendrá que disminuir la cantidad de agua necesaria para el diseño de mezcla.

Los limos y arcillas ocasionaron la variación de humedad entre muestras de canteras, ya que el tamizado en el caso de la piedra, disminuyo el tamaño de las partículas que tenían mayor cantidad de poros y por lo tanto mayor retención de agua.

\section{Absorción de los materiales}

En la tabla 1, se observa que la cantera de Sama presentó valores de absorción de 1,85\%, siendo la de mayor absorción para el agregado fino, las canteras Arunta II, Jonas I, Arunta y Magollo presentaron valores de $1,27 \%, 1,50 \%, 1,62 \%$ y 1,70 \% respectivamente, siendo la cantera de Arunta II la de menor absorción. Respecto al agregado grueso, en la etapa de absorción se obtuvieron valores de 2,25 \% para la cantera Arunta II, la cantera Arunta de 1,40 \% y 1,45\% para las canteras Jonas I y las canteras Magollo y Sama tienen 1,55 \% respectivamente, clasificándose con una absorción de agua baja por encontrarse en el rango de 0, $5 \%-3,0 \%$. Se puede observar que en las canteras estudiadas y la propuesta, presentan rangos aceptables, esto beneficia a los diseños de mezclas, reduciendo la cantidad de agua a añadir, además de tener en cuenta que la humedad contenida en el material aportando cierta cantidad que permitirán reducir los costos. La absorción y el contenido de humedad definen la cantidad exacta de agua que necesita un diseño de mezcla para obtener un concreto con la resistencia requerida. La capacidad de absorción influye directamente en el grado de alteración que podría sufrir el agregado; asimismo la cantidad de vacíos con los poros, con las fisuras y la permeabilidad, son elementos que afectan en toda construcción realizada con concreto.

\section{Peso especifico}

Respecto al peso específico, si el material presenta valores elevados, se interpretan como un material de buen comportamiento, sin embargo valores bajos se interpretan como agregados absorbentes y frágiles. El peso específico es la propiedad que logrará la condición de volumen para los diseños de mezcla de concreto, según los análisis, se clasificó en un agregado normal debido a que muestra el rango establecido de $2500 \mathrm{~kg} / \mathrm{m}^{3}$ a $2750 \mathrm{~kg} / \mathrm{m}^{3}$, esta propiedad es muy importante debido a la buena densidad de los materiales podría lograr un concreto mayo o igual denso. Al tener diferentes tamaños de piedra podrán lograr un alto nivel de peso unitario. Según la tabla 1, la clasificación de los agregados es óptimo para la elaboración de concreto.

El peso específico de los materiales ensayados, tanto como los agregados finos y agregados gruesos son óptimos para la utilización en un buen concreto, y se puede realizar los diseños de mezcla de concreto. Por ello la importancia del peso unitario seco suelto y compactado que ayudan 
a convertir los pesos en volúmenes y viceversa. Con la regularidad del peso unitario se determina los posibles cambios bruscos en la granulometría o forma de los agregados.

\section{Peso unitario seco suelto y compactado}

El peso unitario seco suelto y compactado ayudan a convertir los pesos en volúmenes y viceversa, con la regularidad del peso unitario se puede determinar los posibles cambios bruscos en la granulometría o forma de los agregados. El peso unitario compactado suelto (PUCS) del agregado grueso es empleado en el diseño de mezcla, esta propiedad es muy importante para el manejo, transporte y almacenamiento de los agregados cuando están en estado suelto. En ese contexto, los valores obtenidos para el peso unitario suelto seco en el agregado fino fueron; $1,56 \mathrm{~g} / \mathrm{cm}^{3}, 1,66$ $\mathrm{g} / \mathrm{cm}^{3}, 1,71 \mathrm{~g} / \mathrm{cm}^{3}$ y $1,75 \mathrm{~g} / \mathrm{cm}^{3}$ para las canteras Arunta II, Jonas I, Arunta y Magollo respectivamente, mientras tanto en la cantera de propuesta ubicada en Sama resultó $1,62 \mathrm{~g} / \mathrm{cm}^{3}$, calificándose como aceptable. Los valores del agregado grueso en los ensayos fueron; $1,50 \mathrm{~g} / \mathrm{cm}^{3}$, $1,45 \mathrm{~g} / \mathrm{cm}^{3}, 1,33 \mathrm{~g} / \mathrm{cm}^{3}$ y $1,31 \mathrm{~g} / \mathrm{cm}^{3}$ para las canteras Arunta II, Jonas I, Arunta y Magollo respectivamente mientras que para la cantera de sama fue de $1,52 \mathrm{~g} / \mathrm{cm}^{3}$, como se observa en el cuadro comparativo de resultados.

El peso unitario compactado seco en el agregado fino fueron; $1,77 \mathrm{gr} / \mathrm{cm}^{3}, 1,80 \mathrm{gr} / \mathrm{cm}^{3}, 1,92$ $\mathrm{gr} / \mathrm{cm}^{3}$ y $1,93 \mathrm{gr} / \mathrm{cm}^{3}$ para las canteras Arunta II, Jonas I, Arunta y Magollo respectivamente, mientras tanto en la cantera sama se obtuvo $1,79 \mathrm{gr} / \mathrm{cm}^{3}$, calificándose aceptable para el diseño de mezcla.

Una propiedad física en los materiales es el contenido de vacíos compactos, que muestra la cuantía de volumen que ocupan los poros del volumen total. Esta propiedad es el aire o agua contenido dentro de la muestra. Sin embargo, encontramos que la cantidad de vacíos compactado entre estas partículas dentro del agregado exige una mayor cantidad de mortero al momento de diseñar la mezcla, la angularidad incrementa la cantidad de vacíos y para reducir este contenido o cantidad de vacíos en la muestra se debe tener agregados de mayor dimensión con granulométrica optimizada.

\section{Módulo de finura}

Los materiales más finos que pasan el tamiz $\mathrm{N}^{\circ} 200$, en especial el limo y la arcilla, se presentaron en forma de polvo creando un revestimiento en las partículas de los agregados, estas pueden afectar la ligadura de la pasta de cemento y el material de agregado, logrando perjudicar la resistencia mecánica y el proceso de fraguado. Sin embargo, no se evidenció ejemplares de limo o de material arcilloso, luego de operar el agregado fino y la cantidad de limos eliminada, se mantuvieron los valores con una diminuta variación negativa. Cumplieron con el límite máximo requerido tanto en la cantera menor con un 2,8 y en la cantera mayor con un 3,0 .

En el agregado grueso todos los valores cumplen con el límite máximo requerido, en la cantera de Sama hubo un aumento respecto a la muestra debido a la presencia de piedras grandes, por lo tanto, las partículas de tamaño menor contienen una mayor cantidad de finos adheridos en comparación con las de mayor tamaño que han sido desestimadas al realizar el tamizado, solo hasta 
el $1 \%$ para el agregado fino y un 3\% para el agregado grueso, es el porcentaje máximo de contenido de finos que deben de tener los agregados, superando este límite perjudicaría la resistencia final del concreto. No se tuvo la necesidad de determinar la proporción relativa del contenido de polvo nocivo, o material arcilloso porque no existe en los agregados finos gracias a la granulometría de la arena fina.

\section{Diseño de mezclas}

Respecto al diseño de mezcla no es efectiva en su totalidad debido a que existen canteras que tienen piedras de $3 / 4$ " en forma de canto rodado y otras la obtienen de la piedra triturada a la medida del mercado $\left(1 / 2^{\prime \prime}-3 / 4^{\prime \prime}-3 / 8^{\prime \prime}-1^{\prime \prime}\right)$, por tal razón parecería correcto la proporción de los agregados en ciertos tipos de diseño de mezclas como de ser el caso de un f'c=210 kg/cm2, para esos tipos de agregados pueden ser aceptables ambos tipos como el canto rodado y la chancada (triturada), en cuanto a la calidad de la arena se apreciaron a través de los ensayos que todas muestran buen comportamiento al momento de la realización del concreto, para diferenciar la resistencia se realizó el rompimiento de briquetas para determinar con exactitud si es óptimo para el uso constructivo y comparar a su vez la dosificación obtenida en la cantera de Sama, dado que es necesario un concreto más durable para que resistan eventos sísmicos, y se debe de considerar buenos agregados por ser una zona vulnerable ya que frente a estos eventos se debe de realizar un adecuado encofrado en la infraestructura para que la consistencia sea mejor y evitar la cangrejera que puede ser ocasionada por la piedra de $3 / 4 "$ por lo que debilitaría el elemento estructural y que el canto rodado no permite una mejor fluidez del concreto, en cuanto a la piedra chancada permite que el concreto trabaje de manera eficiente dando un buen comportamiento a la estructura.

En cuanto al diseño de mezcla podemos resaltar que los agregados extraídos de las canteras de Tacna son óptimos para la elaboración de mezclas de concreto con fines constructivos, resaltando que los agregados provenientes de las canteras Arunta, Jonas I y Magollo presentan piedras chancadas de $3 / 4$ " y arena gruesa de buena calidad, siendo estas demostradas al momento de realizar la rupturas de probetas en el laboratorio, en cuanto a la cantera Arunta II presenta también piedras de $3 / 4 "$ pero estas son de canto rodado lo cual mostraron baja resistencia al momento de realizar las rupturas de probetas; sin embargo la cantera de Sama mostro resistencias aceptables al igual que las canteras Arunta, Jonas I y Magollo.

\section{Conclusiones}

Las caracterizaciones de los agregados de las principales canteras de la provincia de Tacna, son óptimas para su uso en obras de construcción; Arunta (agregados, usados en obras de edificaciones y viales), Arunta II (agregados, usados en obras de edificaciones), Cantera Jonas I (agregados, usados en obras de edificaciones y mantenimientos viales), Cantera Magollo (agregados usados para obras de saneamiento y mantenimiento viales).

Los agregados ubicados en Sama, cumplen con los requisitos de la Norma Técnica Peruana (NTP 400.037) y que se consideran aptas para su uso en las obras de construcción respecto a Contenido de humedad (arena $=0,77 \%$, piedra= 0,54 \%), absorción (arena= 1,85\%, piedra= 1,55 \%), 
peso específico (arena= 2,42 $\mathrm{gr} / \mathrm{cm} 3$, piedra $=2,48 \mathrm{gr} / \mathrm{cm} 3$ ), peso unitario suelto (arena= 1,62 $\mathrm{gr} / \mathrm{cm} 3$, piedra $=1,52 \mathrm{gr} / \mathrm{cm} 3$ ), peso unitario compactado (arena= $1,79 \mathrm{gr} / \mathrm{cm} 3$, piedra $=1,69 \mathrm{gr} / \mathrm{cm} 3$ ) y tamaño máximo nominal (piedra=3/4").

Las principales características de la cantera propuesta ubicada en el distrito de Sama, reveló óptimas condiciones para la elaboración de diseño de mezcla para obras de construcción ya que en el análisis destacan parámetros adecuados para su explotación a 7días (promedio de 160,20 $\mathrm{kg} / \mathrm{cm} 2$ ), 14 días (promedio de 195,42 kg/cm2) y 28 días (promedio de 319,61kg/cm2).

Los diseños de mezclas obtenidos mediante el Método $A C l 211$, para $f^{\prime} c=210 \mathrm{~kg} / \mathrm{cm} 2$ con los agregados de las principales canteras de la provincia de Tacna existentes, evidenciaron que todas clasifican en los márgenes establecidos por la NTP, y son similares a las canteras existentes, obteniéndose resistencias a 7 días (promedio de 154,21 kg/cm2) 14 días (promedio de 193,35 $\mathrm{kg} / \mathrm{cm} 2$ ) y 28 días (promedio de $318,81 \mathrm{~kg} / \mathrm{cm} 2$ ).

\section{Referencias Bibliográficas}

Chanini, A. (2015). Fabricación y evaluación de concreto de alta resistencia usando aditivo superplastificante y sílice con cemento Portland Tipo IP en la ciudad de Tacna.

Espinoza, B., \& Mabel, C. (2011). Concreto (hormigón) con Cemento Pórtland Puzolánico tipo ip atlas de resistencias tempranas con la tecnología, sika viscocrete $20 \mathrm{HE}$.

Mamani, L., \& Salinas, R. (2015). Módulo estático de elasticidad del concreto en base a su resistencia a la compresión $\mathrm{Fc}=280 \mathrm{Kg} / \mathrm{cm} 2$, realizado con agregados de la cantera Arunta. Tacna.

Pacheco, C., \& Castillo, V. (2017). Influencia de las Características de los Agregados de las Canteras del Sector el milagro - huanchaco en un diseño de mezcla de concreto. Trujillo.

Piraban, C., \& Farfan, R. (2018). Caracterización Mineralógica y Física de los Agregados de la Cantera Rodeb y Acopios, Aplicada a Concretos y Filtros.

Arévalo.w. (2016). Influencia del agua de mar tratada, a través de un destilador solar, en las propiedades físicas y mecánicas del concreto para la resistencia de 3000 PSI y 4000 PSI, (Tesis). Universidad de Cartagena, Colombia.

Diaz, B., Rios, N., Murga, K., Y Robles, L. (2014). Influencia del agua potable, rio y mar en la resistencia a compresión de un concreto convencional no estructurado, para la construcción de aceras en la ciudad de Trujillo, (Investigación). Universidad Privada del Norte. Trujillo, Perú.

Abanto Castillo, F. (2009). Tecnología del concreto (Teoría y Problemas) (Vol. 2da. edición). Lima: San Marcos E.I.R.L.

339.034, N.T.P. (2008). Método de ensayo normalizado para la determinación de la resistencia a la compresión, en muestras cilíndricas. Lima. 